\title{
Cambios de la proporcionalidad corporal durante el crecimiento en edades adolescentes
}

\author{
M.I. MAESTRE LÓPEZ, M.D. CABAÑAS ARMESILLA, A. HERRERO DE LUCAS. \\ Departamento de Anatomía y Embriología Humana II. \\ Facultad de Medicina, Universidad Complutense de Madrid, España.
}

\begin{abstract}
Resumen
La adolescencia es una etapa crítica de la ontogenia caracterizada por importantes cambios en la velocidad de crecimiento de los tejidos que afectan al tamaño, forma y composición corporal, lo que origina profundas diferencias alométricas entre los individuos según el sexo y estadio madurativo. El presente estudio aborda la valoración del grado de madurez, como índice de salud del adolescente, en función de los índices $Z$ del modelo estándar de proporcionalidad corporal conocido como «Phantom», que representa al promedio poblacional adulto. Aplicando el método antropométrico, se analiza una muestra de 360 adolescentes, entre 10 y 15 años y se encuentra que hacia edades más avanzadas los índices $Z$ de las distintas variables antropométricas se aproximan mejor a los valores del Phantom, hallándose diferencias en la progresión de dichos índices según el sexo, por lo que se debate la adecuación de éste modelo a la interpretación de la realidad cambiante del cuerpo humano.
\end{abstract}

Palabras clave: cineantropometría, phantom, proporcionalidad, alometría, ontogenia, adolescencia.

\section{Introducción}

La curva que representa el crecimiento humano muestra períodos de crecimiento rápido, que determinan fases críticas en las que el proceso resulta más sensible a cualquier incidencia, según los estudios de Tanner entre 1984 y 1996. La adolescencia es una etapa crítica de la ontogenia caracterizada por importantes cambios en la velocidad de crecimiento de los diferentes tejidos y estructuras que afectan al tamaño, forma y

\section{Correspondencia:}

Departamento de Anatomía y Embriología Humana II. Facultad de Medicina, Universidad Complutense de Madrid, España.

E-mails autores por orden según el artículo: imaestre1@yahoo.es, lolacaba@med.ucm.es, aherrero@med.ucm.es. composición corporal. Estas diferencias aparecen igualmente entre sexos, correspondiendo un inicio del estirón puberal más temprano en mujeres que en varones. Aún dentro del mismo rango de edad y sexo, cada individuo alcanza su pico máximo de crecimiento en distintos momentos. Esto se traduce en que, desde una perspectiva ontogénica, existen profundas diferencias alométricas entre los individuos según el sexo y estadío madurativo, debido a que cada tipo de tejido posee su propio modelo y velocidad de crecimiento.

El estudio de los cambios en las proporciones corporales es abordado desde la cineantropometría, empleando el método antropométrico, a través de diversas estrategias, entre las que destaca el modelo estándar de proporcionalidad antropométrica o modelo «Phantom», propuesto por Ross y Wilson en 1974, como aproximación al promedio poblacional, unisexuado, adulto, interracial, 
correctamente nutrido con un grado medio de actividad física.

Dado que la evaluación de los procesos de crecimiento y maduración es un componente fundamental en la vigilancia de la salud del adolescente, el presente estudio aborda la posibilidad de valorar el grado de madurez, en función de los índices $\mathrm{Z}$ del modelo estándar de proporcionalidad antropométrica, a partir de su mayor o menor aproximación al promedio poblacional adulto.

\section{Métodos}

Se ha realizado un estudio controlado, transversal no aleatorio de tipo observacional y analítico sobre una muestra de 368 escolares de edades comprendidas entre los 10 y 15 años, clasificados por sexo y edad, según se muestra en la tabla 1.

Se ha procedido a la aplicación del método antropométrico, para la obtención de medidas antropométricas directas como peso, talla, longitudes, perímetros, pliegues y diámetros empleando para ello material preciso y homologado y siguiendo las recomendaciones del International Working Group of Kinanthropometry (I.S.A.K.) y el Grupo Español de Cineantropometría (G.R.E.C.).

Se ha empleado la estrategia del Phantom para calcular los valores de los índices $\mathrm{Z}$ de las variables antropométricas según la fórmula propuesta por Ross y Wilson (1974) (Fórmula 1) cuyo significado implica el aumento o disminución proporcional de la variable según que $\mathrm{Z}$ sea positivo o negativo (Fig.1).

$$
Z=\frac{1}{S}\left(L \cdot\left(\frac{h_{\text {phantom }}}{h}\right)^{d}-p_{\text {phantom }}\right)
$$

Fórmula 1: Fórmula para el cálculo del índice Z del modelo Phantom. (Ross y Wilson, 1974).

Siendo:

$\mathrm{Z}=$ índice $\mathrm{Z}$ de la medida estudiada.

$\mathrm{S}=$ desviación típica del Phantom relativo a la misma variable.

$\mathrm{L}=$ medida del sujeto de estudio.

$\mathrm{h}=$ talla del sujeto de estudio.

$170.18=$ constante (talla estándar).

$\mathrm{P}=$ valor medio del Phantom.

$\mathrm{d}=$ exponente: 1 para medidas lineales $(\mathrm{L})$.

2 para medidas de superficie $\left(\mathrm{L}^{2}\right)$.

3 para medidas de masa $\left(\mathrm{L}^{3}\right)$.

Para la expresión gráfica de los resultados se han empleado gráficos de dispersión con puntos de datos que relacionan los valores del índice $\mathrm{Z}$ calculado, representado sobre el eje de abcisas, con la variable antropométrica a la que está referido dicho índice, reflejada en el eje de ordenadas. La unión de los puntos entre sí construye los diferentes perfiles de proporcionalidad correspondientes a varones y mujeres de cada edad para establecer la comparación con el modelo adulto estándar promedio (Fig. 2).

\begin{tabular}{|l|l|l|}
\hline Edad (años) & Niños & Niñas \\
\hline 10 & 43 & 14 \\
\hline 11 & 44 & 14 \\
\hline 12 & 71 & 14 \\
\hline 13 & 33 & 18 \\
\hline 14 & 47 & 15 \\
\hline 15 & 39 & 16 \\
\hline Total & 277 & 91 \\
\hline
\end{tabular}

Tabla 1. Distribución muestral por edad y sexo 


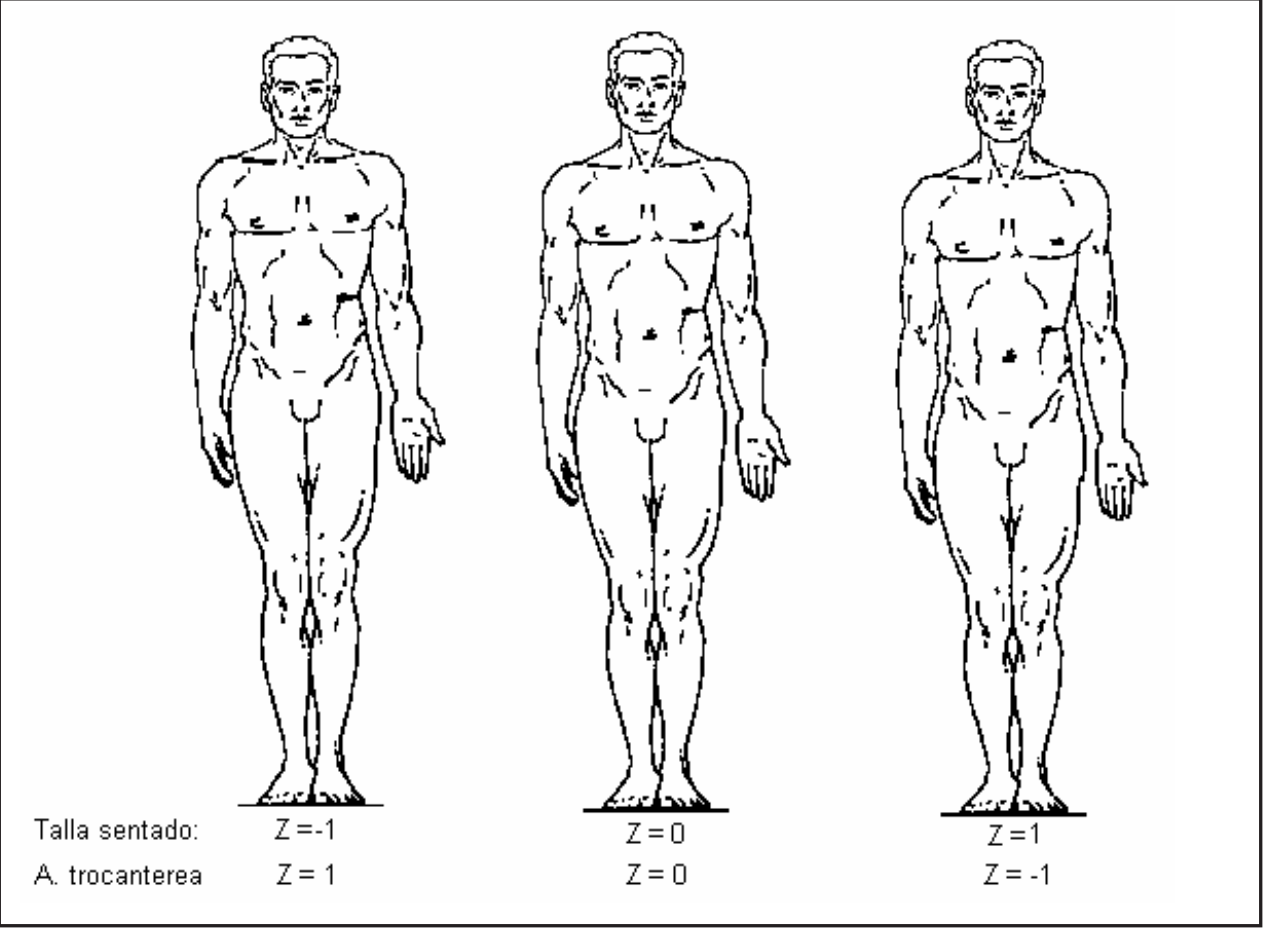

Figura 1. Representación interpretativa de los valores del índice $\mathrm{Z}$ igual y distintos de cero

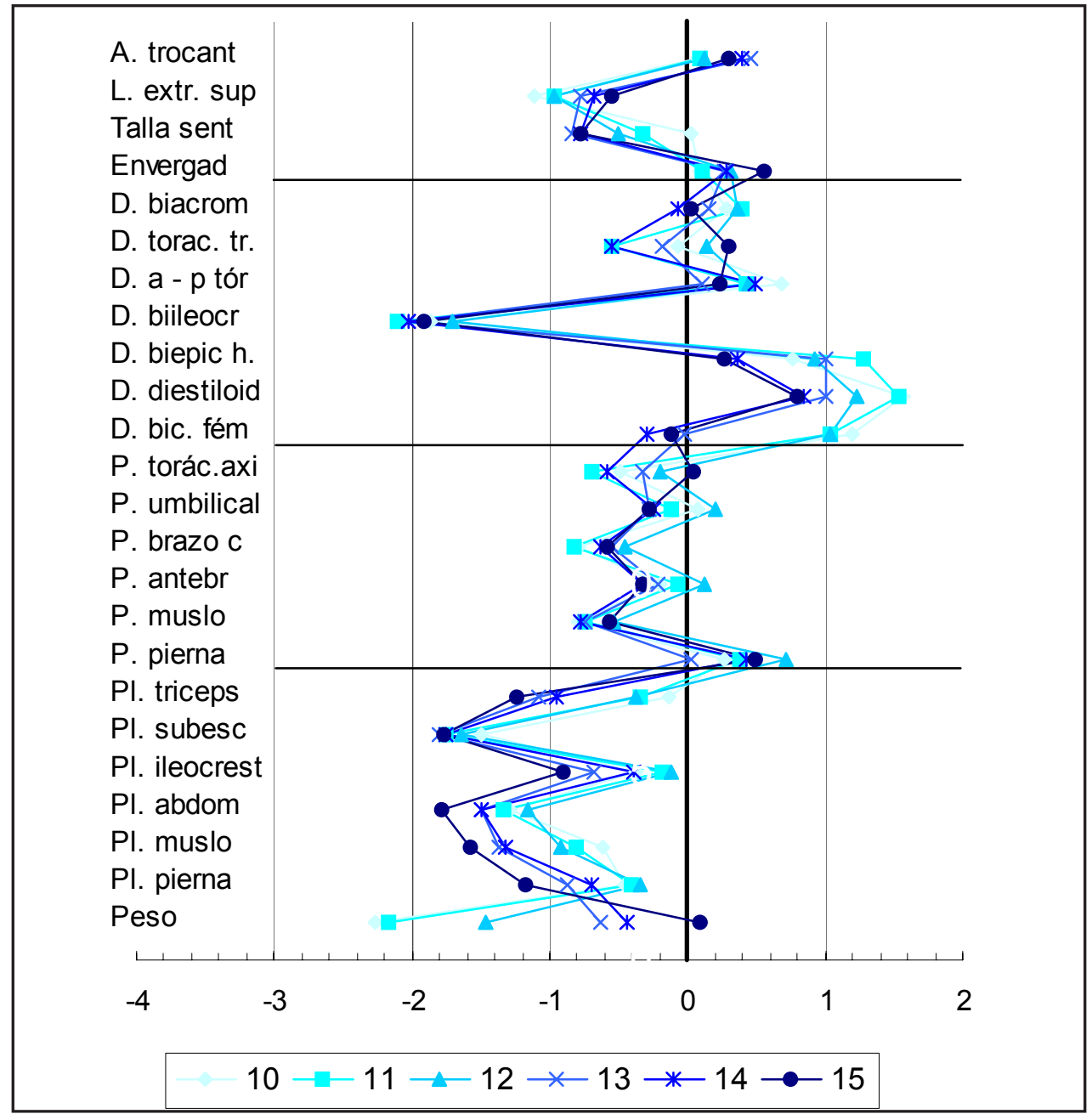

Figura 2. Perfiles de proporcionalidad de varones adolescentes entre 10 y 15 años 
En el presente estudio se propone la valoración del grado de madurez del adolescente en función de los índices Z del modelo estándar de proporcionalidad. La sistemática se basa en que, dado que el modelo estándar es un modelo para adultos, el mayor parecido a este modelo de las distintas componentes de la proporcionalidad, esto es, valores cercanos a cero para la variable $\mathrm{Z}$, indicaría un mayor grado de madurez. A su vez pretende analizar la validez del modelo para estudiar muestras alejadas del promedio poblacional adulto.

\section{Resultados y discusión}

Los valores del índice $\mathrm{Z}$ para varones (Fig.2) y mujeres (Fig.3) adolescentes entre 10 y 15 años, hallados para las 24 variables seleccionadas según el perfil antropométrico completo definido por el I.S.A.K, muestra cómo las proporciones de estas variables se modifican interanualmente para ir desde valores alejados del modelo estándar en adolescentes tempranos hacia proporciones más ajustadas al modelo promedio adulto en adolescentes tardíos, con la excepción de determinadas variables cuya proporcionalidad es característica del sexo, tales como: el diámetro biepicondíleo del húmero, que en varones muestra una proporcionalidad dos unidades de $\mathrm{Z}$ más positiva que las alumnas; o el pliegue del muslo, para el que las mujeres tienden a ser, en su proporcionalidad, una unidad y media más positiva que la de los varones.

Los resultados obtenidos de las tendencias de la proporcionalidad para las distintas variables se pueden resumir de la siguiente forma. Las longitudes y alturas son las variables proporcionalmente más cercanas al modelo adulto para todas las edades. Los diámetros mantienen valores de proporcionalidad superiores al modelo estándar en edades adolescentes tempranas e inferiores en edades tardías. La proporcionalidad de los perímetros es menor de cero en edades

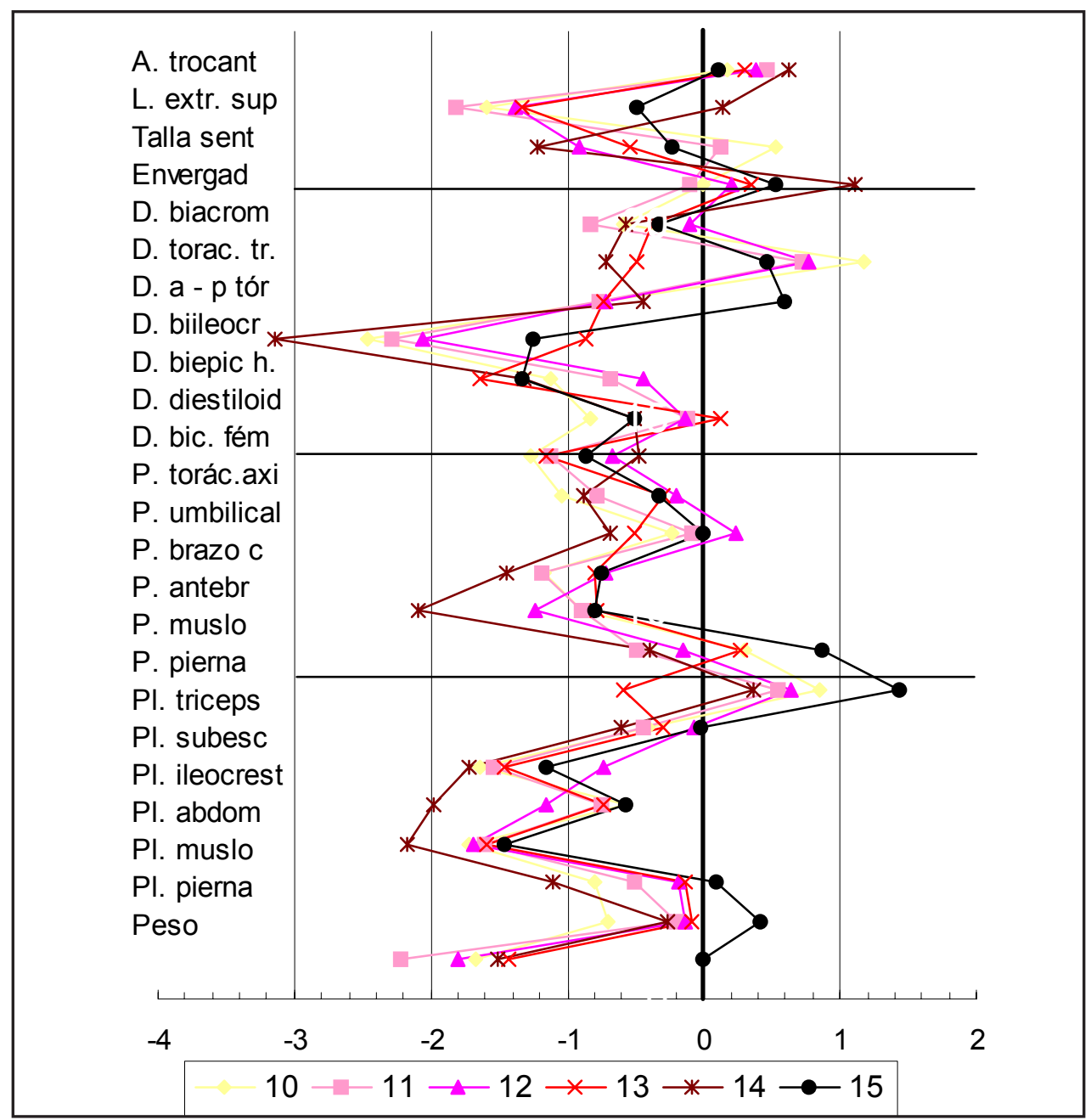

Figura 3. Perfiles de proporcionalidad de mujeres adolescentes entre 10 y 15 años 
adolescentes tempranas y tendentes a cero para edades tardías. La proporcionalidad de los pliegues se mantiene por debajo de los valores estándar para todas las edades; en niñas para todas las edades se muestran por encima de los valores de los varones.

Estos resultados muestran cómo la variación de la proporcionalidad de las variables antropométricas se modifica interanualmente para ir desde valores alejados en adolescentes tempranos hacia proporciones más ajustadas al modelo de referencia en adolescentes tardíos, en distinta magnitud y sentido en función del grupo de variables estudiadas (Fig. 4). Los cambios en las proporciones de estas variables serían propios del proceso de variación interanual o crecimiento de los individuos en la muestra estudiada. Por ello, la variabilidad en estas proporciones puede ser características del proceso ontogénico.

La estrategia del Phantom puede constituir así un método útil en la valoración del grado de madurez en estudios comparativos de crecimiento, que permite completar los estudios de proporcionalidad con otros aspectos interrelacionados desde el punto de vista cineantropométrico, tales como los pliegues como estimadores de la masa grasa, que experimentan mayores cambios en las adolescentes femeninas y las medidas transversales como estimadores de la componente músculo-esquelético, más variables en los adolescentes masculinos.

Sin embargo de su propia definición como modelo estándar unisexuado (Fig.5), que representa el adulto promedio se desprende su inadecuación al modelo promedio real de grupos de edades

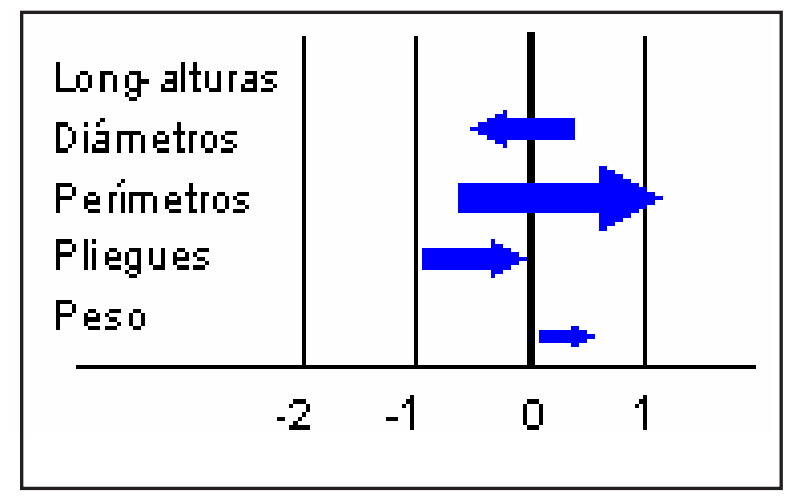

Figura 4 (arriba). Magnitud relativa y sentido de los cambios experimentados por las proporciones cineantropométricas durante el crecimiento del adolescente extremas de la vida, y se revela como escasamente discriminatorio de las diferencias entre individuos debidas al sexo, y otros factores. En concreto en las edades adolescentes destacan dos dificultades al aplicar el modelo adulto al estudio de las proporciones: por un lado, las variaciones alométricas del crecimiento dificultan el estudio de la proporcionalidad al no distinguir el origen ontogénico o ambiental en la diferencia de un índice; por otra parte, como la correlación de la mayor parte de las variables con la edad no es lineal, es difícil predecir el sentido en el que se desplazarán los índices de proporcionalidad.

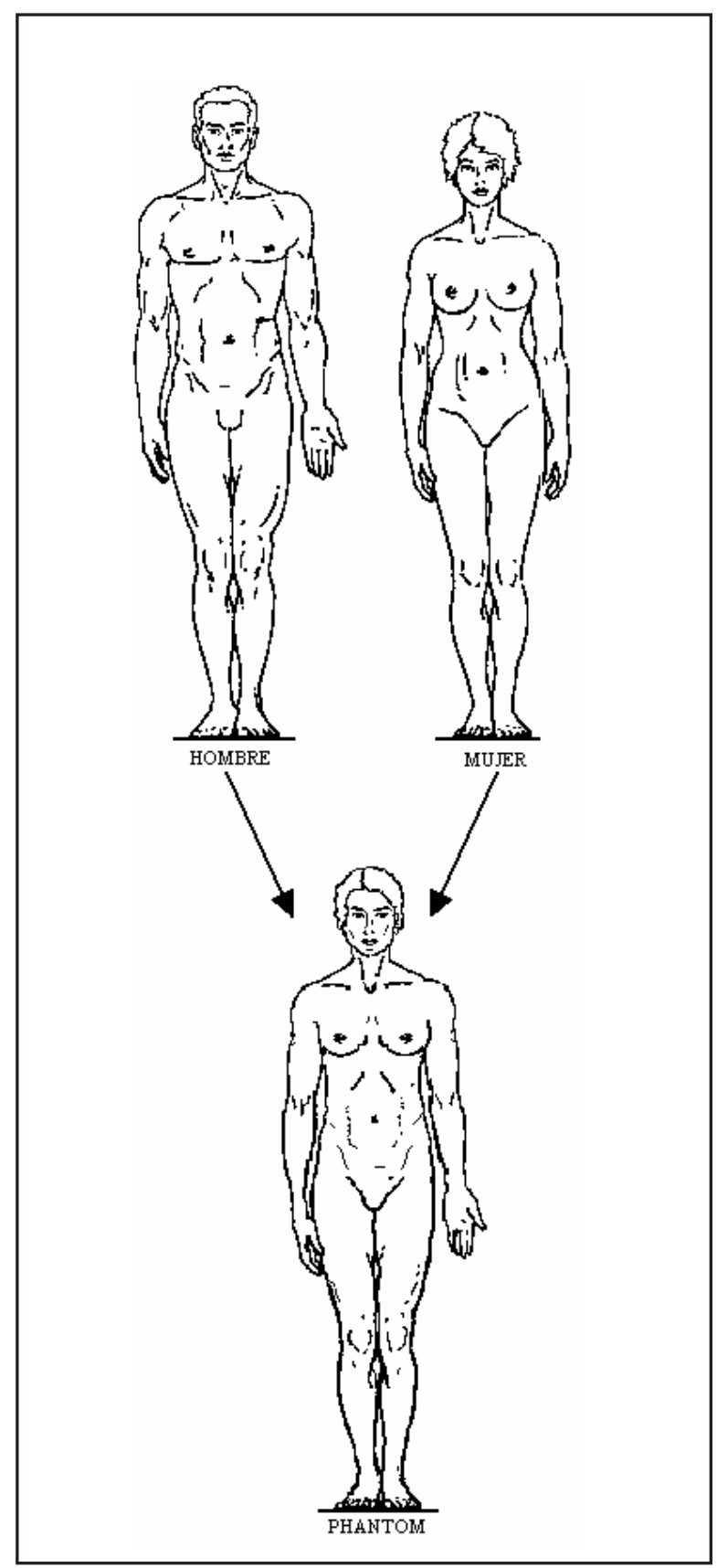

Figura 5 (derecha). Representación de modelo estándar de proporcionalidad adulto, unisexuado 


\section{Conclusiones}

1. Las alturas y longitudes son las variables antropométricas proporcionalmente más cercanas al modelo Phantom para todas las edades. Los diámetros mantienen valores superiores en edades adolescentes tempranas e inferiores en edades adolescentes tardías. 2. La proporcionalidad de las distintas variables con la edad tiende hacia el modelo Phantom, en niños y niñas, con la excepción del diámetro biepicondíleo del húmero que en varones tiende a ser superior al hallado en mujeres, o el pliegue del muslo en el que las mujeres se muestra superior a la de los varones. 3. La estrategia del modelo Phantom permite estudiar las proporciones corporales como índice de maduración en los estudios de crecimiento al menos para el rango de edades estudiado. Sin embargo es necesario adaptarla a la realidad cambiante del cuerpo humano en función de la edad, sexo y otros factores para poder llevar a cabo una mejor vigilancia de su nivel de salud.

\section{Bibliografía}

1. Aragonés, M.T.; Cabañas, M.D; Casajus, J.A.; Rodríguez, F. (1993) Protocolo de medidas antropométricas. (1993) Grupo Español de Cineantropometría. GREC (Ed.) Manual de Cineantropometría. Monografías FEMEDE. Pp. 3566.

2. Durning, J. V., y Womersey, J. (1974): «Body Fat Assessed from Total Body Density and its Estimation from Skinfolds Thickness: Measurements on 4B1 Men and Women Aged from 16 to 72 Years». British Journal Nutrition, 32: 7797.
3. Esparza, F. (1990) Determinación del somatotipo y composición corporal durante el crecimiento. En: Niño, Adolescente y Deporte: «Ortopedia y Traumatología». Monografía FEMEDE I, 2: 44-55.

4. Lapieza, M.G.; Nuviala, R.J.; Castillo, M.C.; Giner, A. (1996) «Estudio morfológico y de porporcinalidad enana población de niñas deportistas». Archivos de Medicina del Deporte. Vol XI. No 41. pp 29-34.

5. Maestre, M.I. (2004) Análisis cineantropométrico del crecimiento en deportistas adolescentes. Tesis doctoral. Facultad de Medicina. Universidad Complutense de Madrid.

6. Pacheco, J.L. (1993) La proporcionalidad corporal. Manual de cineantropometría. Grupo Español de Cineantropometría GREC (Ed). Monografías FEMEDE.pp. 95-112.Pamplona.

7. Ross, W.D. (1985). The Phantom stratagem for proportional growth assessment: questions and answers. Humanbiol., 16: 153-167

8. Ross, W.D.; Bailey, D.A.; Weese, C.H. (1977). Proportionality in interpretation of longitudinal metabolic function data on boys. Frontiers of Activity and Child Health, pp 225-235.

9. Tanner, J.M. (1977). Analysis and classification of physique. Human biology. An introduction to human evolution, variation, growth and ecology, pp. 361-385.

10. Tanner, J.M. (1986). El hombre antes del hombre. El crecimiento físico desde la concepción a la madurez. Ed. Fondo de Cultura Económica de México, 14-118.

11. Shepard, R.J.; LaBarre, R.; Jequier, J.C.; Levallee, H.; Rajic, M.; Volle, M. (1985). The unisex phantom, sexual dimorphism and proportional growth assessment. Amer. J. Phys. Anthrop., 67, 4:403-412.

12. Zamora, S.; Pérez-Llamas, F.; Bouzas, J.C. (2001). Tratado de Nutrición pediátrica. Universidad de Santiago de Compostela. 